\title{
Evidências sobre o isolamento social em pacientes com exsudato em feridas neoplásicas: revisão integrativa*
}

\author{
Evidence about the social isolation in patients with exudate in \\ neoplastic wounds: integrative review
}

\author{
Willian Alves dos Santos ${ }^{1} \bullet$ Patricia dos Santos Claro Fuly ${ }^{2}$ - Mauro Leonardo Salvador Caldeira dos Santos ${ }^{3} \bullet$ \\ Marise Dutra Souto ${ }^{4}$ Caroline Marques Reis ${ }^{5}$ - Luiza de Lima Beretta ${ }^{6}$
}

\begin{abstract}
RESUMO
Objetiva-se identificar as evidências científicas sobre o isolamento social em pacientes com feridas neoplásicas exsudativas. Revisão integrativa realizada nas bases de dados da Biblioteca Virtual de Saúde (BVS) e PubMed: Literatura Latino Americana e do Caribe em Ciências da Saúde (LILACS), Medical Literature Analysis and Retrieval Sistem on-line (MEDLINE) e Cochrane Database of Systematic Reviews (COCHRANE) com a questão norteadora: quais são as evidências científicas sobre o isolamento social em pacientes com feridas neoplásicas exsudativas? Utilizou-se estratégia PICO com recorte temporal de 2002 a 2014, nos idiomas português, inglês e espanhol. As informações foram tabuladas em programa Microsoft Excel ${ }^{\circledR}$ e processadas a partir da média aritmética descritiva simples, frequência absoluta e relativa. Identificaram-se 288 artigos em sua totalidade. Após aplicação dos critérios de elegibilidade, foram selecionadas 23 evidências científicas avaliadas a partir da categoria: os principais aspectos psicossociais prejudicados pelo exsudato em pacientes com feridas neoplásicas. Concluí-se que o exsudato é um dos sintomas que interfere negativamente nos aspectos psicossociais do paciente com feridas neoplásicas, causando alteração da imagem corporal, perda dos limites físicos, ansiedade e depressão, fatores que favorecem diretamente o isolamento social.
\end{abstract}

Palavras-chave: Cuidados Paliativos; Enfermagem Oncológica; Ferimentos e Lesões; Exsudatos e Transudatos; Isolamento Social.

\section{ABSTRACT}

The aim is to identify the scientific evidence on social isolation in patients with exudative neoplastic wounds. An integrative literature review conducted in the Virtual Health Library databases (VHS) and PubMed: Literature Latin American and Caribbean Health Sciences (LILACS), Medical Literature Analysis and Retrieval System online (MEDLINE) and Cochrane Database of Systematic Reviews (COCHRANE), with the guiding question: what are the scientific evidences about the social isolation in patients with exudative neoplastic wounds? We used a PICO strategy with a temporal cut from 2002 to 2014, in Portuguese, English and Spanish languages. The information was tabulated in Microsoft Excel ${ }^{\circledR}$ program and processed from simple descriptive arithmetic mean, absolute and relative frequency. It was identified 288 articles in their entirety. After applying the eligibility criteria, was selected 23 scientific evidence evaluated from the category: the main psychosocial aspects impaired by exudate in patients with neoplastic wounds. It concludes that exudate is one of the symptoms that negatively interferes the psychosocial aspects of the patient with neoplastic wounds, causing alterations in body image, loss of physical limits, anxiety and depression, factors that directly favor social isolation.

Keywords: Palliative Care; Oncology Nursing; Wounds and Injuries; Exudates and Transudates; Social Isolation.

\section{NOTA}

${ }^{1}$ Enfermeiro. Mestre em Ciências do Cuidado em Saúde da Escola de Enfermagem Aurora de Afonso Costa - Universidade Federal Fluminense (EEAAC/UFF). Membro do Grupo Interdisciplinar de Estudos e Pesquisas em Oncologia (GIEPO). Niterói (RJ), Brasil.Email: willian.allves@hotmail.com.

${ }^{2}$ Enfermeira. Doutora em Enfermagem. Professora Adjunta do Departamento de Enfermagem Médico - Cirúrgica da Escola de Enfermagem Aurora de Afonso Costa - Universidade Federal Fluminense (EEAAC/UFF).Vice-chefe do Grupo Interdisciplinar de Estudos e Pesquisas em Oncologia (GIEPO). Niterói (RJ), Brasil. E-mail: claropatricia@yahoo.com.br.

${ }^{3}$ Enfermeiro. Doutor em Enfermagem. Professor do Departamento de Enfermagem Médico-Cirúrgica da Escola de Enfermagem Aurora de Afonso Costa - Universidade Federal Fluminense EEAAC/UFF. Chefe do Grupo Interdisciplinar de Estudos e Pesquisas em Oncologia (GIEPO). Niterói (RJ), Brasil. E-mail: mcaleo@uol.com.br.

${ }^{4}$ Enfermeira. Doutora em Enfermagem. Coordenadora de Projeto de Desenvolvimento do Instituto Nacional de Câncer (INCA). Rio de Janeiro (RJ), Brasil. E-mail: mariseds@ superig.com.br.

${ }^{5}$ Acadêmica do $6^{\circ}$ período de Enfermagem da Escola de Enfermagem Aurora de Afonso Costa - Universidade Federal Fluminense (EEAAC/UFF). Membro do Grupo Interdisciplinar de Estudos e Pesquisas em Oncologia (GIEPO). Niterói (RJ), Brasil. E-mail: reismarques@oi.com.br.

${ }^{6}$ Enfermeira. Mestranda em Ciência do Cuidado em Saúde da Escola de Enfermagem Aurora de Afonso Costa - Universidade Federal Fluminense (EEAAC/UFF). Membro do Grupo Interdisciplinar de Estudos e Pesquisas em Oncologia (GIEPO). Niterói (RJ), Brasil. Email: luizaaberetta@gmail.com

*Manuscrito extraído da dissertação de mestrado intitulada: Associação entre odor, exsudato e isolamento social em pacientes com feridas neoplásicas: um estudo transversal. Universidade Federal Fluminense. 2016. 


\section{INTRODUÇÃO}

As feridas neoplásicas são responsáveis por acometer cerca de $5 \%$ a $10 \%$ dos pacientes com câncer, presenciada, frequentemente, nos últimos seis a doze meses de vida. Geralmente, essas afecções estão ligadas à imprudência do paciente e a sua demora em procurar um auxílio de saúde, bem como ao diagnóstico tardio da doença. 0 tratamento é efetuado majoritariamente de forma paliativa, a fim de minimizar sinais e sintomas, prevendo a melhora na qualidade de vida ${ }^{1}$.

Sob o ponto de vista fisiopatológico, essas lesões originamse a partir da infiltração do tumor, por implantação acidental de células malignas na pele durante o procedimento cirúrgico, diagnóstico ou pela metástase do tumor primário nas composições da pele. Há quebra do tegumento e, como resultado, há danos no local da ferida por meio da combinação da perda da vascularidade, crescimento proliferativo e ulceração ${ }^{2-3}$

Tal lesão apresenta importantes consequências fisiológicas e psicológicas. Destacam-se as de cunho fisiológico: dor, odor fétido, sangramento, infecção e o exsudato. Essas mudanças físicas costumam ser determinantes no que tange as questões psicológicas, gerando mudanças na imagem corporal, estigma, depressão, ansiedade e isolamento social, fatorem que comprometem o bem-estar mental e emocional do paciente 3 .

0 exsudato é o sintoma que está intimamente relacionado às lesões neoplásicas, responsável por afetar cerca de $14,6 \%$ dos pacientes com essa afecção, caracterizando-se por ser de difícil controle e como um grande desafio para o paciente, cuidadores e profissionais de saúde ${ }^{4-5}$. Seu desenvolvimento ocorre por meio da hiperpermeabilidade do tumor ao fibrinogênio e plasma, liberação de fator de permeabilidade vascular e a contaminação da superfície do tumor por bactérias anaeróbias, resultando em grande quantidade de exsudato fibroso por meio de ativação de proteases que degeneram o tecido necrótico levando a sua liquefação $0^{4-8}$.

A perda dos limites físicos é transmitida pela presença da lesão e pelo extravasamento do exsudato derivado da saturação do curativo, o que resulta na necessidade de mudanças frequentes de roupas e na realização de curativos volumosos. Esses fatores favorecem o isolamento social, já que os pacientes passam mais tempo confinados em sua residência a fim de evitar análise e comentários negativos que danifiquem a sua identidade pessoal ${ }^{5}$. Desse modo, a avaliação psicológica do paciente com foco em estratégias de enfrentamento é observada como aspecto relevante de uma avaliação clínica ${ }^{7}$.

$\mathrm{O}$ isolamento social é definido como um estado em que o indivíduo carece de sentimento de pertencimento social, não tendo envolvimento com pessoas, possuindo número mínimo de contatos sociais, sendo deficientes em qualidade ${ }^{8}$. A produção excessiva de exsudato pela lesão faz necessária a troca constante de curativo e de roupas que, por sua vez, afetam diretamente o comportamento social e psicológico do paciente, já que o demasiado tempo destinado a essas atividades fazem o paciente se isolar progressivamente do mundo exterior. Além disso, o vazamento de secreção em locais de convívio social faz gerar sentimento de constrangimento, ansiedade, depressão e estresse. Para minimizar o constrangimento causado por esse e outros sinais e sintomas é necessária uma gestão adequada das feridas neoplásicas no âmbito da atenção oncológica ${ }^{3-5-9}$, considerando assim, a demanda psicológica e social do paciente e seus familiares e/ou cuidador informal na dinâmica do cuidado integralizado.

Tendo em vista a exsudação como fator que prejudica a vida social dos pacientes com feridas neoplásicas, este estudo teve como objetivo identificar as evidências científicas sobre o isolamento social em pacientes com feridas neoplásicas exsudativas.

\section{MÉTODO}

Trata-se de uma revisão integrativa de literatura. Tal método é implementado com a finalidade de sintetizar, de modo abrangente e sistemática, resultados de pesquisas de diversas metodologias sobre determinado tema, fornecendo informações amplas sobre determinado tema ${ }^{10}$.

A pesquisa em questão foi construída em 6 etapas: elaboração da pergunta norteadora; busca ou amostragem na literatura; coleta de dados; análise crítica dos estudos incluídos; discussão dos resultados e apresentação da revisão integrativa ${ }^{10}$.

Realizou-se a investigação a partir de levantamento bibliográfico nas seguintes bases de dados da Biblioteca Virtual de Saúde (BVS) e PubMed: Literatura Latino Americana e do Caribe em Ciências da Saúde (LILACS), Medical Literature Analysis and Retrieval Sistem on-line (MEDLINE) e Cochrane Database of Systematic Reviews (COCHRANE), embasada pela seguinte questão norteadora: quais são as evidências científicas sobre o isolamento social em pacientes com feridas neoplásicas exsudativas?

Para elaboração da questão norteadora foi utilizada a estratégia PICO, que representa o acrômio para $(P=$ Paciente ou Problema, $\mathrm{I}$ = Intervenção, $\mathrm{C}=$ Comparação ou controle, $\mathrm{O}=$ Outcomes ou desfechos ${ }^{11}$. Ressalta-se que para a realização deste estudo foi considerado $\mathrm{P}$ = pacientes com feridas neoplásicas, $\mathrm{I}$ = exsudato e $\mathrm{O}=$ isolamento social (PIO).

A busca foi realizada pelos cruzamentos dos Descritores em Ciências da Saúde (DeCs): exsudatos e transudatos; isolamento social e por Medical Subject Headings Mesh Terms: social isolation, exudates and transudates bem como as palavras-chave e keywords: feridas neoplásicas, feridas tumorais, psychological factors; psychosocial aspects; malignant wound; fungating wound; malignant fungating wound. Como não há termos controlados nacionais e internacionais específicos para feridas neoplásicas, realizou-se o cruzamento entre descritores e as palavras-chave, conectados pelo o operador booleano AND.

As etapas para seleção de artigos científicos realizaram-se a partir da adoção dos critérios de elegibilidade. Os critérios de inclusão foram: textos completos disponíveis online apresentando desenhos de estudos (experimentais, quase experimentais, observacionais e de revisão), com recorte temporal de 2002 a 15 de novembro de 2014, nos idiomas português, inglês e espanhol com aderência ao tema. Foram excluídos os estudos que apresentaram desenho experimental com animais, 
envolvendo crianças e adolescentes em sua amostra de pesquisa e que retratasse de lesões oriundas de radiodermite. A pesquisa nas bases de dados foi realizada em novembro de 2014 .

Salienta-se que o estabelecimento do recorte temporal baseou-se pelo fato de em 2002 a Organização Mundial de Saúde ter revisado o primeiro conceito de cuidados paliativos definido em 1990, que passou a destacar a relevância da prevenção e do alívio do sofrimento para o alcance da qualidade de vida dos pacientes e seus familiares. Como a ferida neoplásica tem seu cuidado geralmente direcionado à paliação, verificou-se a necessidade de identificar todas as evidências científicas a partir desse ano.

Após a seleção dos registros científicos, realizou-se a caracterização dos estudos segundo país de pesquisa, periódico, base de dados indexadora/ano de publicação, tipo de estudo e nível de evidência por meio da proposta de Melnyk e FineoutOverholt ${ }^{12}$.

As informações foram tabuladas em programa Microsoft Excel ${ }^{\oplus}$ e processadas a partir de estratégia de medida de tendência central com a média aritmética descritiva simples, frequência absoluta e relativa. Além disso, realizou-se o mapeamento nos artigos selecionados a fim de encontrar a interferência do exsudato nos aspectos psicossociais dos pacientes com feridas neoplásicas.

\section{RESULTADOS}

Foram identificados 288 artigos científicos em sua totalidade, sendo que após aplicação dos critérios de inclusão e exclusão, foram selecionados 25 estudos. Após a leitura minuciosa dos artigos, restringiu-se para esta pesquisa 23 evidências científicas sobre o eixo temático, como pode ser observado no fluxograma 1.

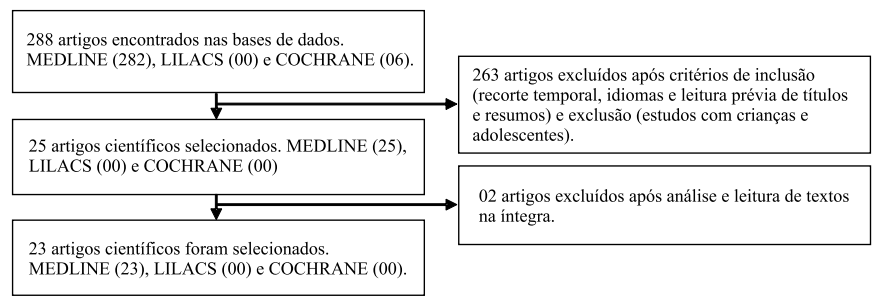

Figura 1. Fluxograma esquemático da estratégia para seleção dos artigos.

Fonte: dados da pesquisa dos autores.

Dentre os artigos selecionados, 95,65\% foram indexados na MEDLINE e 4,34\% na Cochrane, demonstrando que não houve produção nacional comportando o acervo. Sobre o nível de evidência, foi possível inferir que $56,52 \%$ da amostra se enquadraram no nível de evidência $V$, seguido do nível de evidência VI (43,47\%).

No que tange os países de pesquisa, constatou-se que houve maior produção científica sobre o tema na Inglaterra (34,78\%), seguido da Austrália (21,73\%), Suíça (17,39\%), EUA (8,69\%), Taiwan (9\%), Dinamarca, Twain, França e Suécia, cada uma comportando $(4,34 \%)$ das publicações. Desse modo, é evidente que a maior parte das produções e elaborações de pesquisas sobre as feridas neoplásicas ocorreu na Europa (65,21\%).

Após análise dos artigos, observou-se que 52,17\% da amostra apresentaram como desenho de estudo a revisão de literatura em detrimento às seguintes metodologias: fenomenologia (26,08\%), exploratório (8,69\%), descritivo (4,34\%), revisão sistemática (4,34\%) e observacional (4,34\%).

Em relação ao ano de publicação, notou-se que foram obtidos majoritariamente artigos publicados em 2009 com percentual de 21,73\%, seguido dos anos: 2015 (4,34\%); 2014 (4,34\%); 2013 (17,39\%); 2012 (8,69\%); 2010 (13,04\%); 2007 (8,69\%); 2006 (4,34\%) 2005 (13,04\%) e 2002 (4,34\%);

Os periódicos que apresentaram maior taxa de publicação sobre feridas neoplásicas foram: Journal Wond Care comportando $37,78 \%$ (8) da amostra, seguido de International Wound Journal, European Journal of Oncology Nursing e Journal Clinical Nursing com 8,69\% (2) cada um. Já as La Biblioteca Cochrane Plus; Journal of Nursing; Journal Advances Nursing; Seminars Oncology Nursing; Current Opinion Support Palliative Care; Nursing Times; British Journal of Community Nursing; International Journal Palliative Nursing e Support Care Cancer cada um com 4,34\% (1).

Os aspectos psicossociais afetados pelo exsudato em pacientes com feridas neoplásicas são diversas, destacando-se majoritariamente a alteração da imagem corporal (86,25\%) e interação social com a rede familiar prejudicada $(60,86 \%)$.

Observou-se no acervo bibliográfico selecionado determinados instrumentos que possibilitam a mensuração da quantidade de exsudação da lesão e sua taxa de vazamento como facilitadores do cuidado ao paciente oncológico com ferida neoplásica, dentre eles destacam-se: Malignant Fungating Wound Assessment Tool; Treatment Evaluation by LE Roux's (TELER System); Wound Symptoms Self-Assessment Chart (WoSSAC); Pressure Ulcer Scale for Healing (PUSH); Schulz Malignant Fungating Wound Assessment Tool; Toronto Symptom Assessment System for Wounds.

Não foi identificada a utilização de instrumentos ou escalas que permitem a mensuração do isolamento social relacionado ao exsudato dessas lesões nos estudos selecionados.

\section{DISCUSSÃO}

Os resultados obtidos por meio deste estudo foram organizados em uma categoria para discussão dos achados: os principais aspectos psicossociais prejudicados pelo exsudato em pacientes com feridas neoplásicas.

\section{Categoria 1: Os principais aspectos psicossociais prejudicados pelo exsudato em pacientes com feridas neoplásicas.}

Um dos maiores problemas identificados no cenário clínico oncológico sobre feridas neoplásicas é a gestão dos sintomas. É afirmado que essa área é considerada como um grande desafio tanto para os pacientes, quanto para os cuidadores informais e profissionais de saúde. Os sintomas mais comuns presentes neste contexto são de mau odor, exsudato excessivo, dor, sangramento e prurido(8-13-15). Em um estudo prospectivo, notou-se que a dor 
Quadro 1. Distribuição dos estudos incluídos na revisão integrativa

\begin{tabular}{|c|c|c|c|c|c|}
\hline Título & $\begin{array}{l}\text { País de } \\
\text { pesquisa }\end{array}$ & Periódico & $\begin{array}{l}\text { Base de dados/ } \\
\text { Ano }\end{array}$ & Tipo de estudo & $\begin{array}{l}\text { Nível de } \\
\text { evidência }\end{array}$ \\
\hline $\begin{array}{l}\text { An intense and unforgettable experience: the } \\
\text { lived experience of malignant wounds from } \\
\text { the perspectives of patients, caregivers and } \\
\text { nurses. }\end{array}$ & Austrália & Int Woun J. & MEDLINE 2010 & Fenomenológico & $\mathrm{VI}$ \\
\hline $\begin{array}{l}\text { Assessment and management of fungating } \\
\text { wounds. }\end{array}$ & Inglaterra & J Wound Care & MEDLINE 2005 & $\begin{array}{l}\text { Revisão de } \\
\text { literatura }\end{array}$ & V \\
\hline $\begin{array}{l}\text { Care of chronic wounds in palliative care and } \\
\text { end-of-life patients. }\end{array}$ & EUA & Int Woun J. & MEDLINE 2010 & $\begin{array}{l}\text { Revisão de } \\
\text { literatura }\end{array}$ & V \\
\hline $\begin{array}{l}\text { Caring for a loved one with a malignant } \\
\text { fungating wound. }\end{array}$ & Suíça & $\begin{array}{l}\text { Support Care } \\
\text { Cancer. }\end{array}$ & MEDLINE 2012 & Fenomenológico & $\mathrm{VI}$ \\
\hline $\begin{array}{l}\text { Coping with an exulcerated breast carcinoma: } \\
\text { an interpretative phenomenological study }\end{array}$ & Suiça & J Wound Care. & MEDLINE 2013 & Fenomenológico & $\mathrm{VI}$ \\
\hline $\begin{array}{l}\text { Malignant fungating wounds: a survey of } \\
\text { nurses' clinical practice in Switzerland. }\end{array}$ & Suíça & $\begin{array}{l}\text { Eur J Oncol } \\
\text { Nurs. }\end{array}$ & MEDLINE 2009 & Descritivo & $\mathrm{VI}$ \\
\hline $\begin{array}{l}\text { Malignant fungating wounds: an analysis of } \\
\text { the lived experience. }\end{array}$ & Inglaterra & $\begin{array}{l}\text { Int J Palliat } \\
\text { Nurs. }\end{array}$ & MEDLINE 2007 & Fenomenológico & $\mathrm{VI}$ \\
\hline $\begin{array}{l}\text { Malignant fungating wounds: assessment and } \\
\text { management. }\end{array}$ & Inglaterra & $\begin{array}{l}\text { Br J Community } \\
\text { Nurs. }\end{array}$ & MEDLINE 2002 & $\begin{array}{l}\text { Revisão de } \\
\text { literatura }\end{array}$ & V \\
\hline $\begin{array}{l}\text { Malignant fungating wounds: key symptoms } \\
\text { and psychosocial issues. }\end{array}$ & Austrália & J Wound Care. & MEDLINE 2009 & $\begin{array}{l}\text { Revisão de } \\
\text { literatura }\end{array}$ & V \\
\hline $\begin{array}{l}\text { Malignant fungating wounds: managing pain, } \\
\text { bleeding and psychosocial issues. }\end{array}$ & Austrália & J Wound Care. & MEDLINE 2009 & $\begin{array}{l}\text { Revisão de } \\
\text { literatura }\end{array}$ & V \\
\hline $\begin{array}{l}\text { Malignant fungating wounds: the meaning of } \\
\text { living in an unbounded body. }\end{array}$ & Suíça & $\begin{array}{l}\text { Eur J Oncol } \\
\text { Nurs }\end{array}$ & MEDLINE 2013 & Fenomenológico & $\mathrm{VI}$ \\
\hline $\begin{array}{l}\text { Malignant wounds in women with breast } \\
\text { cancer: feminine and sexual perspectives. }\end{array}$ & Dinamarca & J Clin Nurs. & MEDLINE 2005 & $\begin{array}{c}\text { Estudo } \\
\text { exploratório }\end{array}$ & $\mathrm{VI}$ \\
\hline $\begin{array}{l}\text { Malignant fungating wounds: epidemiology, } \\
\text { aetiology, presentation and assessment. }\end{array}$ & Austrália & J Wound Care & MEDLINE 2009 & $\begin{array}{l}\text { Revisão de } \\
\text { literatura }\end{array}$ & V \\
\hline $\begin{array}{l}\text { Malignant fungating wounds: managing } \\
\text { malodour and exudate. }\end{array}$ & Austrália & J Wound Care & MEDLINE 2015 & $\begin{array}{l}\text { Revisão de } \\
\text { literatura }\end{array}$ & V \\
\hline $\begin{array}{l}\text { Managing exudate in malignant fungating } \\
\text { wounds and solving problems for patients. }\end{array}$ & Inglaterra & Nursing Times & MEDLINE 2009 & $\begin{array}{l}\text { Revisão de } \\
\text { literatura }\end{array}$ & V \\
\hline $\begin{array}{l}\text { Malignant wound management in advanced } \\
\text { illness: new insights. }\end{array}$ & Inglaterra & $\begin{array}{l}\text { Curr Opin } \\
\text { Support Palliat } \\
\text { Care. }\end{array}$ & MEDLINE 2013 & $\begin{array}{l}\text { Revisão de } \\
\text { literatura }\end{array}$ & V \\
\hline $\begin{array}{l}\text { Management of malignant fungating wounds } \\
\text { in advanced cancer. }\end{array}$ & EUA & $\begin{array}{l}\text { Semin Oncol } \\
\text { Nurs. }\end{array}$ & MEDLINE 2006 & $\begin{array}{l}\text { Revisão de } \\
\text { literatura }\end{array}$ & V \\
\hline $\begin{array}{l}\text { Psychological profile of patients with } \\
\text { neglected malignant wounds: a qualitative } \\
\text { exploratory study. }\end{array}$ & França & J Wound Care. & MEDLINE 2010 & $\begin{array}{l}\text { Estudo } \\
\text { exploratório }\end{array}$ & $\mathrm{VI}$ \\
\hline $\begin{array}{l}\text { Review of patients' experiences with fungating } \\
\text { wounds and associated quality of life. }\end{array}$ & Inglaterra & J Wound Care. & MEDLINE 2013 & $\begin{array}{l}\text { Revisão } \\
\text { sistemática da } \\
\text { literatura }\end{array}$ & V \\
\hline $\begin{array}{l}\text { Symptom burden and quality of life in patients } \\
\text { with malignant fungating wounds. }\end{array}$ & Taiwan & J Adv Nurs. & MEDLINE 2012 & Transversal & $\mathrm{VI}$ \\
\hline $\begin{array}{l}\text { The meaning of living with malodorous } \\
\text { exuding ulcers. }\end{array}$ & Suécia & J Clin Nurs. & MEDLINE 2007 & Fenomenológico & $\mathrm{VI}$ \\
\hline $\begin{array}{l}\text { The management of malodour and exudate in } \\
\text { fungating wounds. }\end{array}$ & Inglaterra & $\begin{array}{l}\text { Journal of } \\
\text { Nursing, }\end{array}$ & MEDLINE 2005 & $\begin{array}{l}\text { Revisão de } \\
\text { literatura }\end{array}$ & V \\
\hline $\begin{array}{l}\text { Topical agents and dressings for fungating } \\
\text { wounds }\end{array}$ & Inglaterra & $\begin{array}{l}\text { La Biblioteca } \\
\text { Cochrane Plus }\end{array}$ & $\begin{array}{l}\text { COCHRANE } \\
2014\end{array}$ & $\begin{array}{l}\text { Revisão de } \\
\text { literatura }\end{array}$ & V \\
\hline
\end{tabular}

Fonte: dados da pesquisa dos autores. 
Quadro 2. Distribuição dos estudos incluídos na revisão integrativa em relação ao periódico

\begin{tabular}{|l|c|c|}
\hline \multicolumn{1}{|c|}{ Periódico } & N & $\%$ \\
\hline Journal Wound Care & 8 & $37,78 \%$ \\
\hline International Wound Journal & 2 & $8,69 \%$ \\
\hline European Journal Oncology Nursing & 2 & $8,69 \%$ \\
\hline Journal Clinical Nursing & 2 & $8,69 \%$ \\
\hline Current Opinion Support Palliative Care & 1 & $4,34 \%$ \\
\hline Nursing Times & 1 & $4,34 \%$ \\
\hline British Journal Community Nursing & 1 & $4,34 \%$ \\
\hline International Journal Palliative Nursing & 1 & $4,34 \%$ \\
\hline Support Care Cancer & 1 & $4,34 \%$ \\
\hline Journal of Nursing & 1 & $4,34 \%$ \\
\hline Journal Advanced Nursing & 1 & $4,34 \%$ \\
\hline Seminars Oncology Nursing & 1 & $4,34 \%$ \\
\hline La Biblioteca Cochrane Plus & 1 & $4,34 \%$ \\
\hline Total & 23 & $100 \%$ \\
\hline
\end{tabular}

Fonte: dados da pesquisa dos autores.

Quadro 3 Distribuição dos aspectos psicossociais prejudicados pelo exsudato em pacientes com feridas neoplásicas

\begin{tabular}{|l|c|c|}
\hline & N & $\%$ \\
\hline Alteração da imagem corporal & 20 & $86,95 \%$ \\
\hline Dificuldade em se vestir normalmente & 17 & $73,91 \%$ \\
\hline $\begin{array}{l}\text { Interação social com a rede familiar } \\
\text { prejudicada }\end{array}$ & 14 & $60,86 \%$ \\
\hline Depressão & 13 & $56,52 \%$ \\
\hline Ansiedade & 12 & $52,17 \%$ \\
\hline Estresse & 11 & $47,82 \%$ \\
\hline Baixa autoestima & 11 & $47,82 \%$ \\
\hline Identidade prejudicada & 11 & $47,82 \%$ \\
\hline Perda dos limites físicos & 7 & $30,43 \%$ \\
\hline Limitação das atividades físicas diárias & 7 & $30,43 \%$ \\
\hline $\begin{array}{l}\text { Interação social com rede de amigos } \\
\text { prejudicada }\end{array}$ & 7 & $30,43 \%$ \\
\hline Sentimento de solidão & 7 & $30,43 \%$ \\
\hline Limitação em frequentar locais públicos & 6 & $26,08 \%$ \\
\hline Constrangimento pessoal & 6 & $26,08 \%$ \\
\hline Total & 23 & $100 \%$ \\
\hline
\end{tabular}

Fonte: dados da pesquisa dos autores.

(31,2\%), odor $(10,4 \%)$, exsudato $(14,6 \%)$, sangramento $(4,2 \%)$ e prurido (5,2\%) estão associados às feridas neoplásicas(4). 0 exsudato está fortemente relacionado a essas lesões, caracterizando-se por ser de difícil controle, sendo um grande desafio para o paciente, cuidadores e profissionais ${ }^{4-5}$.

Tal sintoma desenvolve-se na lesão por meio da hiperpermeabilidade do tumor ao fibrinogênio e plasma e pela contaminação da superfície do tumor por bactérias anaeróbias, resultando em grande quantidade de exsudato fibroso $0^{4-6}$. 0 exsudato se assemelha ao plasma sanguíneo que compreende água, eletrólitos, nutrientes, mediadores inflamatórios, leucócitos, fatores de crescimento, enzimas e produtos residuais. Existem diferenças importantes entre o exsudato de feridas agudas e de feridas que não cicatrizam, ou seja, crônicas. As feridas crônicas, como exemplo, as feridas neoplásicas, contém menos fatores de crescimento e o aumento dos níveis de enzimas proteolíticas e outras substâncias cáusticas que são capazes de degradar o tecido localizado em volta da lesão e aumentar o tamanho da ferida ${ }^{16}$.

O exsudato é considerado como um grande problema entre os pacientes com feridas neoplásicas, pois ocasiona irritação e maceração da pele circundante à ferida. A quantidade excessiva faz com que haja a saturação do curativo levando ao extravasamento de exsudato sujando, assim, as roupas do paciente, o que ocasiona a necessidade troca de roupas recorrentes. Isso acarreta constrangimentos no estilo de vida, em termos de trocas de curativos e roupa adicional corroborando para que alguns pacientes limitem suas atividades diárias se isolando socialmente ${ }^{17}$.

0 fato do exsudato frequentemente vazar nas vestimentas e roupas de cama não é apenas constrangedor e doloroso para o paciente, mas também aumenta significativamente a carga de trabalho do cuidador. Um estudo de revisão destaca que a vida dos pacientes resume-se às diversas trocas de curativos, vazamento de exsudato e sujidade ${ }^{18}$. 0 exsudato frequentemente vaza nas vestimentas, fato considerado vergonhoso para o paciente, pois afeta diretamente a dignidade, a autoestima e a sociabilidade do paciente 1-4-9-17-19. $^{\text {. }}$

Estudos fenomenológicos internacionais divulgam que a produção excessiva de exsudato faz-se necessário a troca constante de curativo e de vestuário, o que afetam diretamente o comportamento social e psicológico do paciente, já que os pacientes passam a se isolar progressivamente do mundo exterior. Além disso, os vazamentos de secreção em locais de convívio social fazem gerar sentimento de vergonha, ansiedade, depressão, estigma e estresse ${ }^{3-5-9}$. Dessa forma, torna-se imprescindível a reflexão por parte da equipe multiprofissional acerca dos anseios psíquicos e sociais na dinâmica do cuidado aos pacientes com feridas neoplásicas exsudativas.

Além disso, esse sintoma também é caracterizado por ocasionar um profundo impacto na qualidade de vida. Estudos de revisão apontam que esse sintoma é relatado como um dos principais problemas para os pacientes com feridas neoplásicas, levando subsequentemente à irritação da pele e maceração. 0 ajuste no vestuário devido ao vazamento é algo que se torna determinante à mudança no estilo de vida, principalmente em termos de frequência mudanças de roupa e vestimentas adicionais. Esses achados acabam limitando as atividades diárias dos pacientes ${ }^{20}$.

Um estudo fenomenológico desenvolvido com nove pacientes com feridas neoplásicas em mama revelou que a lesão e os sintomas oferecem grande constrangimento aos indivíduos, bem como a visibilidade da progressão da doença, da terminalidade e da perda do controle sobre o corpo, que acabam afetando seriamente a vida cotidiana. A dificuldade no ato de se vestir, 
realizar atividades diárias, o grande tempo disponibilizado à realização de curativos e a preocupação do vazamento de odor, exsudato e sangramento pela ferida se caracterizaram por serem fatores extremamente estressantes, que em conjunto, afetam o comportamento social, emergindo sentimento de marginalização, raiva, estigma, solidão e consequentemente influência negativa no bem-estar mental e social do paciente ${ }^{5}$.

A alteração da imagem corporal advindo da ferida e seus sintomas, bem como o impacto do tratamento em termos de curativos e a sensação de falha na apresentação pessoal, resultam na sua busca constante de pretextos de situações sociais. Existe a necessidade de uma rede de apoio social para permitir sucesso na adaptação da imagem corporal alterada, porém para muitos pacientes, o fato do baixo conhecimento sobre a lesão por parte da rede de base social desencadeia redução do apoio, resultando em sentimentos de exclusão e isolamento ${ }^{21-22}$.

O vazamento de exsudato causa muitos problemas aos pacientes. As consequências desse evento e da perda dos limites físicos ocorrem devido à realização de curativos que não são capazes de conter efetivamente o exsudato, gerando frequentes mudanças de roupas, o que aumenta a quantidade de tempo destinado à lavagem de roupas e estratégias para a contenção. Esse evento faz com que os pacientes se refugiem em suas residências, onde se sentem seguros de comentários negativos e análise que danificam a sua identidade. A necessidade de realização de inúmeras trocas de curativos devido aos frequentes vazamentos de exsudato durante o dia são extremamente estressantes, pois geralmente dispende grande tempo para a sua execução, o que reduz significativamente a socialização ${ }^{5}$. Desse modo, isso aponta para a necessidade de avaliação psicológica do paciente, com foco em estratégias de enfrentamento no processo do cuidado e da avaliação clínica7.

Além disso, é extremamente relevante a gestão do exsudato, uma vez que auxilia na diminuição do odor, proteção da pele sadia perilesional, aumento do conforto do paciente e a melhora de sua dignidade e qualidade de vida ${ }^{23}$. Em consonância ao exposto, para haver o manejo adequado das feridas neoplásicas, o odor e o exsudato devem ser considerados, enquanto ao mesmo tempo focando no gerenciamento do desconforto e isolamento resultante dessas condições, devendo o conforto e a qualidade de vida do paciente ser, nesta etapa, priorizados ${ }^{24}$. 0 acesso ao cuidado especializado, curativos modernos e redução dos angustiantes sintomas permitem ao paciente mais conforto, menos angústia e redução do estigma e do isolamento social devido à administração adequada desse sintoma ${ }^{5}$.

Para o controle desses sinais e sintomas, além de realização de curativos adequados a cada particularidade do paciente, é necessário o conhecimento de produtos que visem minimizar os sintomas presentes na lesão. Para o controle de exsudato, utiliza-se comumente o Metronidazol $0,8 \%$, bem como produtos que executam o debridamento enzimático, procedimento recomendado através da utilização de agentes químicos específicos, ou seja, substâncias enzimáticas, tais como: Hidrogel e Hidrocoloide ${ }^{6-25-26}$. Essa técnica pode efetivamente suavizar e remover o tecido necrosado, principal responsável pela produção de exsudato ${ }^{26}$.
O Enfermeiro deve orientar o paciente e familiar e estimular a troca tão logo sujem de secreção a fim de auxiliar no controle do exsudato. Em alguns casos pode ser difícil para o paciente manter a sua própria higiene, de forma independente, devendo o familiar ser orientado sobre a importância de que esta seja executada apropriadamente ${ }^{27-29}$. 0 foco do cuidado às lesões tumorais não é a cicatrização, mas o manejo e controle de sinais e sintomas. Assim, torna-se relevante que os enfermeiros estejam habilitados adequadamente às diversas necessidades da demanda do cuidado a esses pacientes no que tange os aspectos físicos, psicológicos, sociais, espiritual e econômico.

Vale mencionar que os enfermeiros são diagnosticadores no processo de cuidado. Nesse caso, o isolamento social se destaca como sendo um diagnóstico de enfermagem respaldado pela North American Nursing Diagnosis Association's (NANDA), que proporciona ao enfermeiro autonomia de identificá-lo e diagnosticá-lo de acordo com as características, definições e classificações presentes no indivíduo ${ }^{30}$. Destaca-se também que no Catálogo internacional para a prática de Enfermagem (CIPE $\left.{ }^{\oplus}\right)$ tal diagnóstico é evidenciado, destacando-se, assim, as inúmeras ferramentas que podem subsidiar a práxis profissional nesse contexto ${ }^{31}$.

Além disso, a CIPE - Cuidados paliativos para uma morte digna - catálogo com linguagem unificada disponibilizada a fim de nortear a práxis de Enfermagem oncológica em Cuidados Paliativos, o isolamento social é um dos domínios de preocupação do instrumento, destacando a importância da gestão e intervenção do enfermeiro perante esse diagnóstico, contribuindo para melhor qualidade de vida do paciente ${ }^{32}$.

Vale mencionar que há na literatura os sistemas que possibilitam a mensuração da quantidade de exsudato na lesão neoplásica, favorecendo uma ação adequada ao seu controle. O Treatment Evaluation by A Le Roux's Method (TELER Sistem), é um instrumento utilizado na área oncológica que viabiliza a mensuração de vários aspectos ligados à ferida neoplásica. Dentre as metas estão o tratamento, registros de resultados a nível individual e em grupo, como também a mensuração do odor e exsudato. Nesse contexto, a proposta desse sistema em relação ao exsudato é avaliar a quantidade de vazamento pelo curativo em um período de 24 horas $^{33}$.

O Toronto Symptom Assessment System for Wounds busca avaliar sistematicamente o grau de dor e outros sintomas, bem como a angústia associada a todas as classes de feridas. É composto por 10 parâmetros de sintomas que são avaliados individualmente em escalas de avaliação numérica de 11 pontos. Pode ser aplicado na prática clínica para orientar a gestão sintomas relacionadas à ferida ${ }^{4}$. Porém, no âmbito nacional, não há instrumentos validados que favoreçam a avaliação do exsudato e do isolamento social em pacientes com feridas neoplásicas.

\section{CONCLUSÃO}

O exsudato é um dos sintomas que pode favorecer, diretamente, o isolamento social em pacientes com feridas neoplásicas. Observa-se que esse sintoma está relacionado à sensação de vergonha por parte dos pacientes. A quantidade 
excessiva de secreção com a necessidade de realização de curativos volumosos, somado ao fato da sensação eminente de vazamento e às constantes trocas para evitar o vazamento, pode levar a necessidade de mudança na rotina de vida, com consequente impacto psicossocial do paciente culminando no isolamento social.

No que tange a limitação do estudo, verificou-se que não há, até então, pesquisas publicadas na área oncológica abordando o impacto psicossocial dos sintomas em pacientes com feridas neoplásicas no âmbito brasileiro, ressaltando a necessidade de elaboração de pesquisas que contenham significativo nível de evidência no cenário nacional. Além disso, espera-se que este estudo incentive a realização de outras pesquisas que abordem tal eixo temático, pois é determinante multiplicar o conhecimento sobre o assunto aos diversos integrantes da equipe multiprofissional, para assim, garantir uma visão holística ao paciente.

Na equipe multidisciplinar, a enfermagem desempenha papel importantíssimo no manejo e controle de sinais e sintomas dos pacientes com feridas neoplásicas. Além do conhecimento sobre as idiossincrasias dessas lesões e dos produtos adequados ao cuidado e controle do exsudato, é fulcral que o profissional de enfermagem conheça os principais sintomas que essa lesão apresenta e o impacto que eles podem causar ao cotidiano do paciente. Conhecer esse impacto fará toda a diferença na práxis do enfermeiro, pois, assim, poderá atuar de maneira assertiva e holística no cuidado ao paciente com ferida neoplásica, enxergando, além dos sintomas, seus anseios psicológicos e sociais.

\section{REFERÊNCIAS}

1. Aguiar RM, Silva GR. Os cuidados de enfermagem em feridas neoplásicas na assistência de enfermagem. Rev Hospital Universitário Pedro Ernesto. [Internet] 2012 [acesso em 30 de Outubro de 2013]; 11(2):82-88. Disponível em: http:// revista.hupe.uerj.br/detalhe_artigo.asp?id=331.

2. Firmino F. Pacientes portadores de feridas neoplásicas em Serviços de Cuidados Paliativos: contribuições para a elaboração de protocolos de intervenções de enfermagem. Rev Brasileira de Canc. [Internet] 2005 [acesso em 03 de novembro de 2014]; 51(4):347-59. Disponível em: http:// www.inca.gov.br/rbc/n_51/v04/pdf/revisao6.pdf

3. Probst S, Arber A,Trojan A, Faithfull S. Caring for a loved one with a malignant fungating wound. Support care cancer [Internet]. 2012[acesso em 03 de novembro de 2014]; 20(12):3065-3070. Disponível em: http://epubs.surrey. ac.uk/533557/3/caring\%20for\%20loved\%20one.pdf.

4. Maida V, Ennis M, Kuziemsky G, Trozzolo L. Symptoms Associated with Malignant Wounds: A Prospective Case Series. Jour of Pain and Symptom Management. [Internet] 2009; [acesso em 31 de Outubro de 2013] 37(2):20611. Disponível em: http://download.journals.elsevierhealth. com/pdfs/journals/0885-3924/PIIS0885392408002996.pdf.

5. Probst S, Arber A, Faithfull S. Malignant fungating wounds: The meaning of living in an unbounded body. European Journal of Oncol Nurs. [Internet] 2013 [acesso em 03 de novembro de 2014]; 17(1)38-45. Disponível em: http:// linkinghub.elsevier.com/retrieve/pii/S1462-3889(12)00019-1.

6. Gozzo TO, Tahan FP, Andrade M, Nascimento TG, Prado MAS. Ocorrência e manejo de feridas neoplásicas em mulheres com câncer de mama avançado. Esc Anna Nery. [Internet] 2014 [acesso em 15 de abril de 2016]; 18(2)270-76. Disponível em: http://www.scielo.br/pdf/ean/v18n2/1414-8145ean-18-02-0270.pdf.

7. Selby T. Managing exudate in malignant fungating wounds and solving problems for patients. Nursing Times. [Internet] 2009 [acesso em 03 de novembro de 2014]; 105(18):14-7. Disponível em: http://www.ncbi.nlm.nih.gov/ pubmed/19507614.

8. Carri H, Mary AM, Richard B, Jyoti AK, Diane B, Karen HB. Social isolation in older adults who are frequent users of primary care services. Canadian Family Physician. [Internet] 2014 [acesso em 23 de março de 2014]; 60(6)322-29. Disponível em: http://www.cfp.ca/content/60/6/e322.full.pdf+html.

9. Lo SF, Hu W, Hayter M, Chang S, Hsu M, Wu L. Experiences of living with a malignant fungating wound: a qualitative study. J ClinNurs. [Internet] 2008 [acesso em 23 de março de 2014]; 17(20): 2699-708. Disponível em: http://www.ncbi. nlm.nih.gov/pubmed/18808638.

10. Souza MT, Silva MD, Carvalho R. Revisão integrativa: o que é e como fazer. Einstein. [Internet]. 2010 [acesso em 14 mar 2016]; 8(1 pt 1):102-6. Disponível em: http://www. astresmetodologias.com/material/O_que_e_RIL.pdf.

11. Petrolo E, Danski MTR, Mingorance P, Lazzari LSM, Méier MJ, Crozeta K. A prática baseada em evidências como ferramenta para prática profissional do enfermeiro. Cogitare Enfermagem. [Internet] 2009 [acesso em 15 de dezembro de 2015]; 14(4): 760-3. Disponível em: http://www.revenf.bvs. $\mathrm{br} / \mathrm{pdf} / \mathrm{ce} / \mathrm{v} 14 \mathrm{n} 4 / \mathrm{a} 23 \mathrm{v} 14 \mathrm{n} 4 . \mathrm{pdf}$

12. Melnyk BM, Fineout-Overholt E. Making the case for evidence-based practice. In: Melnyk BM, Fineout-Overholt E. Evidence-based practice in nursing and health care. A guide to best e practice. Philadelphia: Lippincout Willian \& Wilkins; 2005.

13. Probst S, Arber A, Faithfull S. Malignant fungating wounds: A survey of nurses' clinical practice in Switzerland. European Journal of Oncol Nurs. [Internet] 2009 [acesso em 23 de março de 2014]; 13(4)295-298. Disponível em: http://www. ejoncologynursing.com/article/S1462-3889(09)00048-9/ fulltext.

14. Dolbeaut S,Flahault C,Baffie A,Fromantin I. Psychological profile of patients with neglected malignant wounds: a qualitative exploratory study. Jour of wound care. [Internet] 2003 [acesso em 03 de novembro de 2014]; 19(12)513-21. Disponível em: http://www.magonlinelibrary. com/doi/abs/10.12968/jowc.2010.19.12.513?url_ ver=Z39.88-2003\&rfr_id=ori:rid:crossref.org\&rfr_dat $=c r_{\text {_ }}$ pub\%3dpubmed.

15. Grocott P. Care of patients with Fungating Malignant wound. Nurs Standv. [Internet] 2007 [acesso em 03 de novembro de 2014]; 21(24)57-8. Disponível em: http://www.ncbi.nlm.nih. gov/pubmed/17345910.

16. Alexander S. Malignant fungating wounds: epidemiology, aetiology, presentation and assessment. Jour of wound care. [Internet] 2009 [acesso em 03 de novembro de 2014]; 
18(7)273-80. Disponível em: http://www.thinktag.it/system/ files/2121/mi412_.pdf?1292080188.

17. Grocott $P$. The palliative management of fungating malignant wounds. Journal of Wound Care. [Internet] 2000 [acesso em 03 de novembro de 2014]; 9(1)4-9. Disponível em: http:// www.ncbi.nlm.nih.gov/pubmed/10827661.

18. Alexandre S. Malignant fungating wounds: key symptoms and psychosocial. Jourofwoundcare. [Internet] 2009 [acesso em 25 de outubro de 2013]; 18(8).325-29. Disponível em: http://www.ncbi.nlm.nih.gov/pubmed/19862870.

19. Lund-Nielsen B, Adamsen L, Gottup F, Rosth M, Tolver A, Kolms HJ. Qualitative bacteriology in malignant wounds - a prospective, randomized, clinical study to compare the effect of honey and silver dressings. Ostomy wound manage. [Internet] 2011 [acesso em]; 57(2)28-36. Disponível em: http://www.ncbi.nlm.nih.gov/pubmed/21904013.

20. Gibson S, Green R. Review of patients' experiences with fungating wounds ad associated quality of life. Jour of wound care. [Internet] 2013 [acesso em 03 de novembro de 2014]; 22(5)265-75. Disponível em: http://www. journalofwoundcare.com/cgibin/go.pl/library/article. cgi?uid=98467; article=JWC_22_5_265_275;format=pdf.

21. Price, B. Assessing altered body image. Jour of Psychiatric and Mental Health Nursing. [Internet] 1995 [acesso em 13 de abril de 2015]; 2(3)169-75. Disponível em: http:// onlinelibrary.wiley.com/doi/10.1111/j.1365-2850.1995. tb00052.x/abstract.:

22. Piggin C, Jones V. Malignant fungating wounds: an analysis of the lived experience. International journal of palliative nursing. [Internet] 2007 [acesso em 03 de novembro de 2014];13(8)384-91. Disponível em: http://www.magonlinelibrary.com/doi/abs/10.12968/ jowc. 2009.18.2.38744? url_ver=Z39.88-2003 \& rfr_ $i d=o r i:$ rid:crossref.org \& rfr_dat=cr_pub\%3dpubmed .

23. Agra G, Fernandes MA, Platel ICS, Freire MEM. Cuidados paliativos ao paciente portador de feridas neoplásicas: uma revisão integrativa da literatura. Rev brasileira de cancerologia. [Internet] 2012 [acesso em 03 de novembro de 2014]; 59(1)95-104. Disponível em: http://www1.inca.gov. br/rbc/n_59/v01/pdf/16-cuidados-paliativos-ao-pacienteportador-de-ferida-neoplasica.pdf.

24. O'Brien C. Malignant wounds: managing odour. Canadian family physician. [Internet] 2012 [acesso em 03 de novembro de 2014]; 58(3)272-74. Disponível em: http://www.ncbi.nlm. nih.gov/pmc/articles/PMC3303648/.

25. Froiland KG. Wound care of the advanced cancer patient. Hematology Oncology Clinics of North America. [Internet] 2002 [acesso em 03 de novembro de 2014]; 16(1)629-39. Disponível em: http://www.ncbi.nlm.nih.gov/ pubmed/12170572.

26. Grocott P, Gethin G, Probst S. Malignant wound management in advanced illness: new insights. CurrOpin Support Palliat Care. [Internet] 2013[acesso em 03 de novembro de 2014]; 7(1):101-5. Disponivel em: http://journals.lww. com/co-supportiveandpalliativecare/pages/articleviewer. px?year $=2013$ \&issue $=03000 \&$ article $=00018 \&$ type $=$ abstract

27. West D. A palliative approach to the management of malodour from malignant fungating tumours. International Jour of Palliative Nurs. [Internet] 2007 [acesso em 03 de novembro de 2014]; 13(3)137-42. Disponível em: http://www.magonlinelibrary.com/doi/abs/10.12968/ ijpn.2007.13.3.23276?url_ver=Z39.88-2003\&rfr_ id=ori:rid:crossref.org\&rfr_dat=cr_pub\%3dpubmed .

28. Hawthorn M. Caring for a patient with a fungating malignant lesion in a hospice setting: reflecting on practice. International Jour of Palliative Nurs. [Internet] 2010 [acesso em 03 de novembro de 2014]; 16(2)70-6. Disponível em:http://www.magonlinelibrary.com/doi/abs/10.12968/ ijpn.2010.16.2.46752?url_ver=Z39.88-2003\&rfr_ id=ori:rid:crossref.org \&rfr_dat=cr_pub\%3dpubmed

29. Harrison T. Introducing a holistic wound dressing. British jour of Nurs.[Internet] 2014 [acesso em 03 de novembro de 2014]; 22(12)14-17. Disponível em: http://www.magonlinelibrary. com/doi/pdf/10.12968/bjon.2013.22.Sup12a.14

30. Carpenito-Moyet ப. Social Isolation. In: CARPENITO-MOYET, Lynda Juall. Nursing diagnosis: application to clinical practice. 12 ed. Estados Unidos da América: The point. 2008, p. 634.

31. CIPE. Cuidados paliativos para uma morte digna - Catálogo da classificação internacional para a prática de Enfermagem. Portugal: Ordem dos Enfermeiros. 2010.

32. CIPE 2.0. Classificação internacional para a prática de Enfermagem. 2a.ed. São Paulo: Algol. 2011.

33. Grocott P. Developing a tool for researching fungating wounds. World wide wounds. [Internet] 2001 [acesso em 03 de novembro de 2014]; . Disponível em: http://www. worldwidewounds.com/2001/july/Grocott/FungatingWounds.html\#TELER-system. 